\title{
Decoupled polarization dynamics of incoherent waves and bimodal spectral incoherent solitons
}

\author{
A. Fusaro, ${ }^{1}$ J. Garnier, ${ }^{2}$ C. Michel, ${ }^{3}$ G. Xu, ${ }^{4, *}$ J. Fatome, ${ }^{1}$ L. G. Wright, ${ }^{5}$ F. W. Wise, ${ }^{5}$ and A. Picozzi ${ }^{1}$ \\ ${ }^{1}$ Laboratoire Interdisciplinaire Carnot de Bourgogne (ICB), UMR 6303 CNRS-Université Bourgogne Franche-Comté, F-21078 Dijon, France \\ ${ }^{2}$ Laboratoire de Probabilités et Modèles Aléatoires, University Paris Diderot, 75205 Paris Cedex 13, France \\ ${ }^{3}$ Université Nice Sophia Antipolis, CNRS, Laboratoire de Physique de la Matière Condensée, UMR 7336, 06100 Nice, France \\ ${ }^{4}$ Université Lille, CNRS, UMR 8523-PhLAM-Physique des Lasers Atomes et Molécules, F-59000 Lille, France \\ ${ }^{5}$ School of Applied and Engineering Physics, Cornell University, Ithaca, New York 14853, USA \\ *Corresponding author: gang.xu@ircica.univ-lille1.fr
}

Received 23 June 2016; revised 29 July 2016; accepted 1 August 2016; posted 1 August 2016 (Doc. ID 268901); published 23 August 2016

We consider the propagation of strongly incoherent waves in optical fibers in the framework of the vector nonlinear Schrödinger equation (VNLSE) accounting for the Raman effect. On the basis of the wave turbulence theory, we derive a kinetic equation that greatly simplifies the VNLSE and provides deep physical insight into incoherent wave dynamics. When applied to the study of polarization effects, the theory unexpectedly reveals that the linear polarization components of the incoherent wave evolve independently from each other, even in the presence of weak fiber birefringence. When applied to light propagation in bimodal fibers, the theory reveals that the incoherent modal components can be strongly coupled. After a complex transient, the modal components self-organize into a vector spectral incoherent soliton: The two solitons self-trap and propagate with a common velocity in frequency space. () 2016 Optical Society of America

OCIS codes: (190.4370) Nonlinear optics, fibers; (190.5650) Raman effect; (030.1640) Coherence.

http://dx.doi.org/10.1364/OL.41.003992

The propagation of partially coherent nonlinear optical waves is a subject of growing interest in different fields of investigations, such as, e.g., supercontinuum (SC) generation [1-8], lasers [9,10], rogue waves $[11,12]$, or shock waves $[13,14]$. Remarkable phenomena such as optical wave condensation [15,16], or incoherent soliton propagation with inertial $[7,17,18]$, resonant [19], or nonlocal [20] nonlinearities have been discussed in different contexts. A different form of "spectral incoherent soliton" (SIS), which cannot be identified in the temporal domain but solely in the frequency domain, has been also reported in optical fiber systems [21-24].

While incoherent wave propagation has been widely studied in the scalar regime, incoherent phenomena inherently associated with the vector nature of the optical field received much less attention $[7,18,25]$. In this Letter, we study the dynamics of strongly incoherent waves propagating in optical fiber systems in the framework of the vector nonlinear Schrödinger equation (VNLSE) accounting for the Raman effect. We consider the VNLSE to model two different problems: (i) polarization effects in single mode fibers; (ii) light propagation in bimodal optical fibers. A wave turbulence approach of the problem reveals that the dynamics of the incoherent optical wave is dominated by the Raman effect. When applied to polarization effects (i), the theory reveals a decoupled polarization dynamics: At variance with coherent waves where the inhibition of an energy exchange among the polarization components requires a strong birefringence [24], here, the incoherent components evolve independently of each other even in the presence of a weak birefringence. On the other hand, when the theory is applied to bimodal fibers (ii), an unexpected strong coupling among the incoherent modal components arises. In this way, the incoherent waves are shown to self-organize into a novel form of vector SIS (VSIS), which generalizes the previously studied scalar SISs [21-24]. Along this way, our kinetic theory can be extended to study the turbulent dynamics of broadband spatiotemporal spectra in multimode fibers [8], whose originating mechanisms are the subject of growing recent interest $[26,27]$.

In the first part of the Letter, we consider the standard VNLSE that governs the evolution of the polarization components of the optical field. It accounts for both the parallel and orthogonal Raman contributions [3,28,29]:

$$
\partial_{z} A_{j}+\hat{d}_{j} A_{j}=i \gamma\left(\left(1-f_{R}\right) P_{j}+f_{R} \sum_{l=1}^{3} Q_{j}^{(l)}\right)
$$

with

$$
\begin{gathered}
P_{j}=\left(\left|A_{j}\right|^{2}+\frac{2}{3}\left|A_{3-j}\right|^{2}\right) A_{j}+\frac{1}{3} A_{j}^{*} A_{3-j}^{2} \exp \left(i \phi_{j}\right), \\
Q_{j}^{(1)}=\left(R_{\|} *\left|A_{j}\right|^{2}+R_{o} *\left|A_{3-j}\right|^{2}\right) A_{j}, \\
Q_{j}^{(2)}=\left(R_{\perp} *\left(A_{j}^{*} A_{3-j}\right)\right) A_{3-j} \exp \left(i \phi_{j}\right), \\
Q_{j}^{(3)}=\left(R_{\perp} *\left(A_{j} A_{3-j}^{*}\right)\right) A_{3-j},
\end{gathered}
$$


where $A_{j}(t, z) \quad(j=1,2)$ refers to the linear polarization components. The dispersion operator reads $\hat{d}_{j} A_{j}=$ $(-1)^{j+1} \delta \beta_{1} \partial_{t} A_{j}+i \frac{1}{2} \beta_{2, j} \partial_{t t} A_{j}$, where $\delta \beta_{1}=\beta_{1,1}-\beta_{1,2}, \beta_{k, j}$ denoting the $k$ th order dispersion parameter of $A_{j}(z, t)$. The phase mismatch, $\phi_{j}=(-1)^{j} 2 \Delta \beta z$ with $\Delta \beta=\beta_{0,1}-\beta_{0,2}$ accounts for fiber birefringence. The term $P_{j}$ refers to the instantaneous Kerr nonlinearity, while $Q_{j}$ refers to the delayed Raman nonlinearity, the symbol $*$ denoting the convolution product, and $\gamma$ the nonlinear coefficient. We recall that $R_{\|}(t)$ denotes the usual dominant parallel Raman response function [24], while the perturbative orthogonal Raman contribution is $R_{\perp}(t)=H(t) \frac{r}{\tau_{R}} \exp \left(-t / \tau_{R}\right)$ with $\tau_{R}=32 \mathrm{fs}$ and $r \simeq 1 / 15, H(t)$ being the Heaviside function, and $R_{o}(t)=R_{\|}(t)-2 R_{\perp}(t)$.

Usually, the perturbative orthogonal Raman contribution is neglected, $R_{\perp}(t)=0$, so that $R_{o}(t)=R_{\|}(t), Q_{j}^{(2)}=Q_{j}^{(3)}=0$, and Eq. (1) recovers the standard model describing polarization effects in birefringent fibers $[1,2,4,5]$ \{also see Eq. (13.5.2) in [24]\}. In the following we consider strongly incoherent waves, in such a way that dispersion effects dominate nonlinear effects, $L_{d, j} \ll L_{n l}$, where $L_{d, j}=2 t_{c, j}^{2} / \beta_{2, j}$ are the dispersion lengths, $t_{c, j}$ being the time correlations of $A_{j}$, and $L_{n l}=1 /(\gamma P)$ is the nonlinear length, $P=\left\langle\left|A_{1}\right|^{2}\right\rangle+\left\langle\left|A_{2}\right|^{2}\right\rangle$ being the total conserved power.

We report in Fig. 1 the evolution of the spectrum of an incoherent wave obtained by integrating numerically the VNLSE (1). In this example, the incoherent wave is injected along one of the principal axes of the fiber, say $A_{1}$. The initial condition is a partially coherent wave with a Gaussian spectrum and random spectral phases, superimposed on a small noise spectral background. The Gaussian spectral bandwidth is larger than the Raman frequency shift $\sim 13.2 \mathrm{THz}$. We deliberately considered a weakly birefringent regime, $L_{B}=2 \pi / \Delta \beta=L_{n l}$, so that the corresponding nonresonant terms in VNLSE (involving the factor $\sim \exp \left(i \phi_{j}\right)$ ) do not average out as in the strong birefringent regime. Hence, such terms are liable to induce a transfer of energy between $A_{1}$ and $A_{2}$. In contrast, we see in Fig. 1 that there is almost no transfer of energy among the orthogonal polarization components (i.e., the incoherent wave remains polarized along the 1 -axis). In this way, the system behaves essentially as in the scalar case [7]: The spectral evolution of $A_{1}$ is characterized by the generation of a SIS,
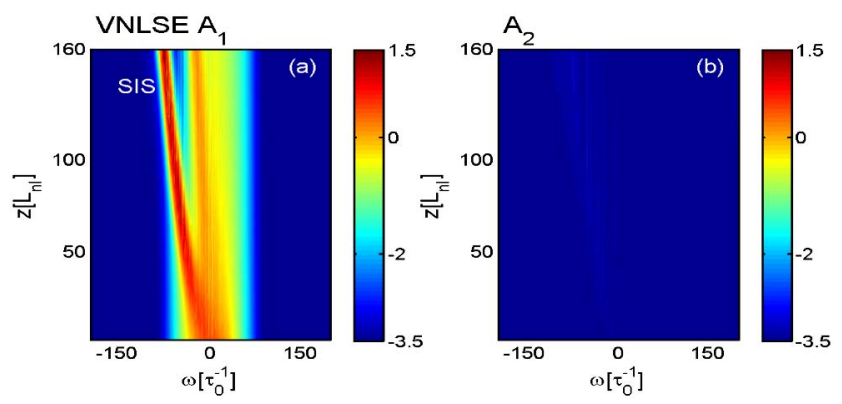

Fig. 1. Polarization dynamics: evolutions of the spectrum of the incoherent orthogonal polarization components (a) $A_{1}$ and (b) $A_{2}$, obtained by solving the VNLSE (1). The incoherent wave is injected along the 1-axis. Despite the presence of a weak birefringence $\left(L_{B}=L_{n l}\right)$, there is almost no transfer of energy to the 2-axis. The system then evolves as in the scalar case: A SIS is generated in the component $A_{1}$ (see the text for parameters). which is continuously red-shifted with a constant spectral velocity during the propagation.

In Fig. 2 the incoherent wave is injected in both polarization components $A_{1}$ and $A_{2}$, with an energy fraction of 0.7 for $A_{1}$ ( 0.3 for $\left.A_{2}\right)$. The initial condition in $A_{1}$ has been set identical to that considered in Fig. 1, so as to easily compare the evolution of $A_{1}$ in the presence of $A_{2}$ (Fig. 2), to the corresponding evolution in the absence of $A_{2}$ (Fig. 1). Such a comparison remarkably reveals that the presence of $A_{2}$ does not affect the spectral evolution of $A_{1}$ : The polarization components evolve independently of each other. Accordingly, the SIS generated in $A_{1}$ does not interact with the SIS that is going to emerge in $A_{2}$; see Fig. 2.

In order to understand the decoupled polarization dynamics, we resort to a statistical description of the incoherent waves based on the wave turbulence theory $[7,30]$. We note that the procedure underlying the derivation of the vector kinetic equations (VKEs) closely follows that of the pure scalar kinetic equation (KE), whose derivation is detailed in Ref. [7]. Assuming that the weakly nonlinear random wave exhibits a stationary statistics, one obtains a closure of the hierarchy of moments equations. The averaged spectra of the polarization components, $\quad\left\langle\tilde{A}_{j}(\omega+\Omega / 2, z) \tilde{A}_{k}^{*}(\omega-\Omega / 2, z)\right\rangle=n_{j}(\omega, z) \delta(\Omega) \delta_{j, k}^{K}$ $\left[\delta_{j, k}^{K}\right.$ denoting the Kronecker symbol], evolve according to the VKE:

$$
\begin{aligned}
\partial_{z} n_{j}(\omega, z)= & \frac{\gamma f_{R}}{\pi} n_{j} \int g_{\|}\left(\omega-\omega_{1}\right) n_{j}\left(\omega_{1}\right) \mathrm{d} \omega_{1} \\
& +\frac{\gamma f_{R}}{\pi} n_{j} \int g_{\perp}\left(\omega-\omega_{1}\right) n_{3-j}\left(\omega_{1}\right) \mathrm{d} \omega_{1},
\end{aligned}
$$

where the spectral gain curves read, $g_{\|}(\omega)=\Im\left[\tilde{R}_{\|}(\omega)\right]$, and $g_{\perp}(\omega)=\mathfrak{I}\left[\tilde{R}_{\perp}(\omega)\right]$. The VKE conserves the total energy during the propagation, $N=\sum_{j} \int n_{j}(\omega, z) \mathrm{d} \omega=$ const. We recall that the VKE is valid in the weakly nonlinear regime, while a higher-order cubic collision term should be included
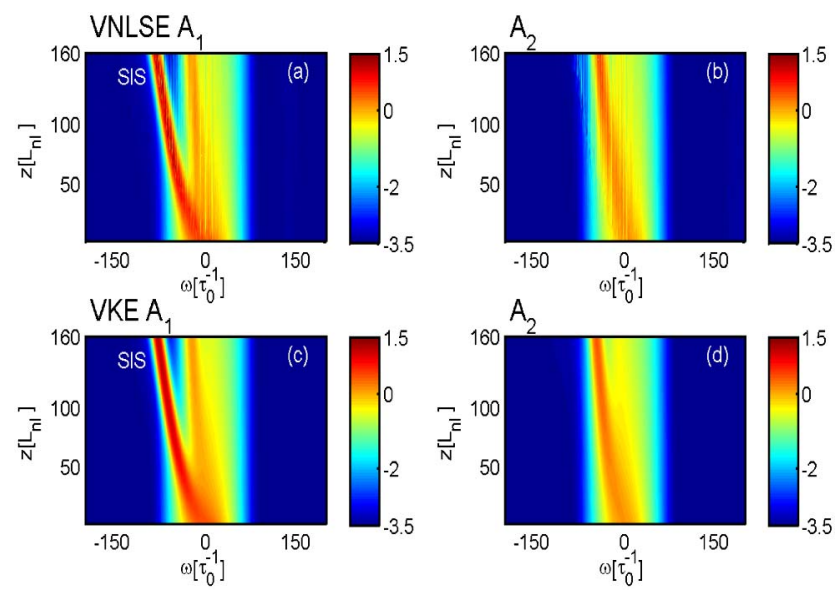

Fig. 2. Polarization dynamics: evolutions of the spectra of the orthogonal polarization components $A_{1}$ (left column) and $A_{2}$ (right column), obtained by solving the VNLSE (1) (first line) and VKE (6) (second line). The initial condition in $A_{1}$ is the same as in Fig. 1: The comparison reveals that $A_{1}$ is not affected by the presence of $A_{2}$. As a result of their decoupled evolutions, the soliton generated in $A_{1}$ refers to a purely scalar SIS. Parameters are $L_{B}=L_{n l}=1.55 \mathrm{~m}$, $\beta_{2,1}=\beta_{2,2}=-20 \mathrm{ps}^{2} / \mathrm{m}$, a "healing time" $\tau_{0}=123 \mathrm{fs}$, and $\delta \beta_{1}=0.04 \tau_{0} / L_{n l}=3.18 \mathrm{fs} / \mathrm{m}$. 
in the KE to describe stronger nonlinear effects [7]. The VKE also assumes statistical stationary fluctuations of the incoherent field, while deviations from stationarity can be described in the framework of a generalized KE combining the above KE with a Vlasov formulation of the problem; see Ref. [31].

We stress the remarkable simplicity of the VKE (6) as compared to the VNLSE (1). First, as in the usual scalar case, both effects of linear dispersion and instantaneous Kerr nonlinearity do not enter the VKE, a property that has been confirmed by several previous works $[7,13,22,23]$. In the vector problem considered here, the cross-phase modulation term (second term in $Q_{j}^{(1)}$ ) that couples the polarization components, is averaged out by the incoherence of the waves. In addition, the nonresonant birefringence terms $\left[Q_{j}^{(2)}\right.$ in Eq. (4)] do not contribute to the KE. This is due to the fact that the terms $Q_{j}^{(2)}$ solely involve correlators of the form, $\left\langle\tilde{A}_{j}^{2}(\omega)\right\rangle(j=1,2)$ : In contrast to the correlators $n_{j}(\omega)$, which are phase-independent, the correlators $\left\langle\tilde{A}_{j}^{2}(\omega)\right\rangle$ vanish because the rapid random phases of $\tilde{A}_{j}(\omega)$ do not cancel out. It turns out that, in contrast to coherent wave propagation, here, the nonresonant terms $Q_{j}^{(2)}$ are averaged out even in the weakly birefringent regime.

The above discussion reveals that $Q_{j}^{(3)}$ in Eq. (5) are the unique terms that contribute to the VKE (6). As a remarkable consequence, whenever one neglects the perturbative orthogonal Raman gain, $g_{\perp}(\omega)$ (see Refs. $[1,2,5]$ ), the VKE reveals that there is no coupling at all between the polarization components $A_{1}$ and $A_{2}$. Numerical simulations confirm this prediction, as revealed by the quantitative agreement between VKE (6) and VNLSE (1) simulations in Figs. 1 and 2, without using adjustable parameters.

Note that, although the polarization components $A_{1,2}$ are incoherent and decorrelated, there is one of the Stokes parameters that does not vanish, $S_{1}=\left\langle\left|A_{1}\right|^{2}\right\rangle-\left\langle\left|A_{2}\right|^{2}\right\rangle$, since there is no energy exchange between $A_{1}$ and $A_{2}$. As a consequence, the degree of polarization of the incoherent beam is conserved during the propagation and does not vanish, $\mathrm{DOP}=S_{1} / P=$ const.

We remark that we have not considered the impact of random birefringence fluctuations [24], which can be neglected provided that their correlation length $\left(L_{c}\right)$ is the larger length scale (i.e., $L_{c} \gg L_{n l}$ ). It is important to note that the analysis reported here is also relevant to rapid birefringence fluctuations (i.e., in the opposite regime $L_{c} \ll L_{d} \ll L_{n l}$ ), where the VNLSE is known to recover Manakov-like equations [32]. We have extended the computation of Ref. [32] by including the complete Raman contribution. The analysis reveals that the corresponding KE keeps the same form as Eq. (6), except that the interaction coefficients are slightly changed by the averaging procedure over fast birefringence fluctuations.

As a consequence of the decoupled polarization dynamics discussed above, the SISs generated in the polarization components $A_{1}$ and $A_{2}$ evolve independently of each other, as illustrated in Fig. 2. In order to study the interaction between SISs, a strong coupling among them is required, a feature that can be found in multimode fibers [6]. For simplicity, we consider here the example of a bimodal fiber in which two linearly polarized waves $A_{1}$ and $A_{2}$ propagate, respectively, along the first $\mathrm{LP}_{01}$ and $\mathrm{LP}_{11}$ modes of the fiber. The general form of the VNLSE governing the evolutions of the modal components $A_{j}(j=1,2)$ can be found in [6] and its structure is analogous to Eq. (1). To see this, let us focus our attention on such terms that originate in the Raman effect. The analysis of the overlap integrals among the two modes reveals that only four distinct terms contribute to the VNLSE, and their structures exactly coincide with the terms $Q_{j}^{(l)}(l=1,2,3)$ reported in Eqs. (3)-(5). More precisely, the terms $Q_{j}^{(l)}$ involved in the modal VNLSE can be recovered from Eqs. (3)-(5) through the substitutions: $R_{o} \rightarrow \kappa_{12} R_{\|}$in $Q_{j}^{(1)} ; R_{\perp} \rightarrow \kappa_{12} R_{\|}$in $Q_{j}^{(2)}$ and $Q_{j}^{(3)}$; and $\gamma \rightarrow\left(n_{2} \omega_{0} / c\right) S_{1111}$ (assuming $S_{1111}=S_{2222}$ for simplicity), where $\kappa_{12}=S_{1212} / S_{1111}\left(S_{\text {plmn }}\right.$ being the overlap integrals; see Eq. (8) in [6]).

As discussed above, through polarization effects, a coupling among the modal components $A_{j}(j=1,2)$ is provided solely by the term $Q_{j}^{(3)}$. This becomes apparent in the modal VKE, which is recovered from Eq. (6) through the substitution: $g_{\perp} \rightarrow \kappa_{12} g_{\|}$. The key observation is that, at variance with the polarization VNLSE (1), the contribution of $Q_{j}^{(3)}$ in the modal VNLSE is no longer a perturbation. Indeed, considering the concrete example of a bimodal fiber of core radius $a=5 \mu \mathrm{m}$, core and cladding refractive indices $n_{c o}=1.4556$, $n_{c l}=1.45$, and pump wavelength $\lambda_{0}=1550 \mathrm{~nm}$, we obtain $\kappa_{12} \simeq 0.626$, which reveals a strong coupling among the modes $A_{1}$ and $A_{2}$. There is also a strong phase mismatch among the modes, $\Delta \beta \simeq 9 \times 10^{3} L_{n l}^{-1}$, so that $Q_{j}^{(2)}$ averages to zero.

The numerical simulations of the modal VNLSE and modal VKE reveal that, contrary to polarization effects, the strong modal coupling is responsible for a significant transfer of energy among the modes, which eventually leads to the spontaneous formation of a VSIS: The SISs that evolve in the two modes $A_{1}$ and $A_{2}$ self-trap and propagate with a common velocity in frequency space; see Fig. 3. We have derived the following analytical vector soliton solution of the VKE:

$$
n_{j}^{s}(\omega)=n_{0}+\left(n_{j, m}^{s}-n_{0}\right) \exp \left[-\log \left(\frac{n_{j, m}^{s}}{n_{0}}\right) \frac{\omega^{2}}{\omega_{0}^{2}}\right],
$$
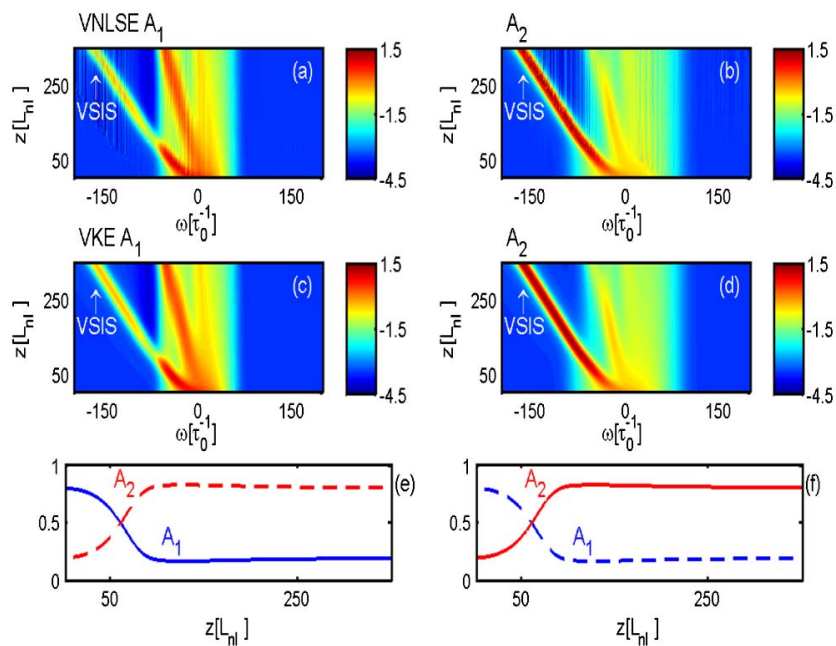

Fig. 3. Bimodal fiber dynamics: evolutions of the spectra of the modal components $A_{1}$ [left column: (a),(c)] and $A_{2}$ [right column: (b),(d)], obtained by solving the modal VNLSE (first line) and modal VKE (second line). Corresponding evolutions of the energies in each mode [third line: (e),(f)]. At variance with polarization effects (Fig. 2), the modal components are strongly coupled: $A_{1}$ and $A_{2}$ spontaneously self-organize into a bimodal VSIS. Parameters are $L_{n l}=1.55 \mathrm{~m}$, $\beta_{2,1}=\beta_{2,2}=-20 \mathrm{ps}^{2} / \mathrm{m}, \tau_{0}=123 \mathrm{fs}$, and $\delta \beta_{1}=5.6 \mathrm{ps} / \mathrm{m}$. 

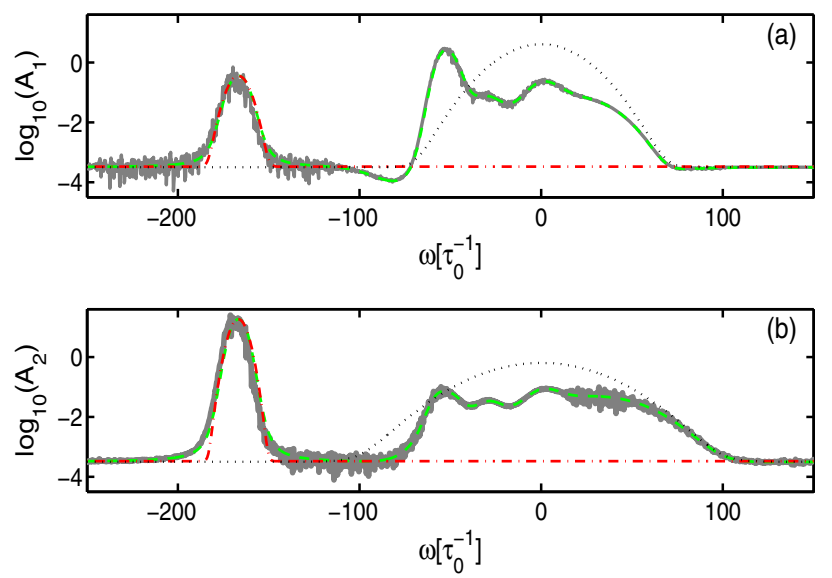

Fig. 4. Bimodal fiber dynamics: spectral profiles of the VSIS corresponding to the numerical simulation reported in Fig. 3 at $z=350 L_{n l}$ : modal VNLSE (gray line); modal VKE (dashed green line); analytical VSIS solution from Eq. (7) (dot-dashed red line), initial condition (dark dashed line). The quantitative agreement between the modal VNLSE, modal VKE, and analytical VSIS solution (7), has been obtained without adjustable parameters.

where $n_{0}$ refers to the constant background noise, and $\omega_{0}=$ $\sqrt{2}\left[-\partial_{\omega} g_{\|}(0)\right]^{-1 / 2}\left[-\int_{0}^{\infty} g_{\|}(\omega) \mathrm{d} \omega\right]^{1 / 2}$ denotes the typical width of $g_{\|}(\omega)$. Note that the soliton maximum amplitudes $n_{j, m}^{s}$ are coupled to each other by the common soliton velocity. We obtained a good agreement between the analytical bimodal SIS solution (7) and the simulations of the VKE and VNLSE, as remarkably illustrated in Fig. 4. Note a minor discrepancy of solution (7) in the tails of the soliton, a feature that can be ascribed to the fact that Eq. (7) refers to an approximation in the vicinity of the soliton peak of a more general form of the soliton solution, a feature that will be discussed elsewhere together with a detailed presentation of the continuous family of multimode SIS solutions.

In summary, we have derived a VKE that greatly simplifies the original VNLSE. At variance with coherent waves, we showed that a weak birefringence leads to a decoupled evolution of the polarization components of the incoherent wave. On the other hand, incoherent waves that propagate in the two modes of a bimodal fiber can be strongly coupled, leading to the spontaneous formation of a VSIS.

Funding. Agence Nationale de la Recherche (ANR) (ANR12-BS04-0011 OPTIROC); Labex ACTION (ANR-11LABX-01-01); European Research Council (ERC) (FP7/ 20072013, 306633).

\section{REFERENCES}

1. S. Coen, A. H. L. Chau, R. Leonhardt, J. D. Harvey, J. C. Knight, W. J. Wadsworth, and P. St. J. Russell, J. Opt. Soc. Am. B 19, 753 (2002). 2. Z. Zhu and T. G. Brown, J. Opt. Soc. Am. B 21, 249 (2004).
3. E. R. Martins, D. H. Spadoti, M. A. Romero, and B.-H. V. Borges, Opt. Express 15, 14335 (2007).

4. B. J. Chick, J. W. M. Chon, and M. Gu, Opt. Express 16, 20099 (2008).

5. H. Tu, Y. Liu, X. Liu, D. Turchinovich, J. Laegsgaard, and S. A. Boppart, Opt. Express 20, 1113 (2012).

6. P. Horak and F. Poletti, Recent Progress in Optical Fiber Research, M. Yasin, S. W. Harun, and H. Arof, eds. (InTech, 2012), pp. 3-25.

7. A. Picozzi, J. Garnier, T. Hansson, P. Suret, S. Randoux, G. Millot, and D. Christodoulides, Phys. Rep. 542, 1 (2014).

8. L. G. Wright, D. N. Christodoulides, and F. W. Wise, Nat. Photonics 9 , 306 (2015).

9. D. V. Churkin, I. V. Kolokolov, E. V. Podivilov, I. D. Vatnik, S. S. Vergeles, I. S. Terekhov, V. V. Lebedev, G. Falkovich, M. A. Nikulin, S. A. Babin, and S. K. Turitsyn, Nat. Commun. 2, 6214 (2015).

10. E. Turitsyna, S. Smirnov, S. Sugavanam, N. Tarasov, X. Shu, S. Babin, E. Podivilov, D. Churkin, G. Falkovich, and S. Turitsyn, Nat. Photonics 7, 783 (2013).

11. N. Akhmediev, B. Kibler, F. Baronio, M. Belic, W. Zhong, Y. Zhang, W. Chang, J.-M. Soto-Crespo, P. Vouzas, P. Grelu, C. Lecaplain, K. Hammani, S. Rica, A. Picozzi, M. Tlidi, K. Panajotov, A. Mussot, A. Bendahmane, P. Szriftgiser, G. Genty, J. Dudley, A. Kudlinski, A. Demircan, U. Morgner, S. Amiraranashvili, C. Bree, G. Steinmeyer, C. Masoller, N. Broderick, A. Runge, M. Erkintalo, S. Residori, U. Bortolozzo, F. Arecchi, S. Wabnitz, C. Tiofack, S. Coulibaly, and M. Taki, J. Opt. 18, 063001 (2016).

12. P. Walczak, S. Randoux, and P. Suret, Phys. Rev. Lett. 114, 143903 (2015).

13. J. Garnier, G. Xu, S. Trillo, and A. Picozzi, Phys. Rev. Lett. 111, 113902 (2013).

14. G. Xu, D. Vocke, D. Faccio, J. Garnier, T. Rogers, S. Trillo, and A. Picozzi, Nat. Commun. 6, 8131 (2015).

15. P. Aschieri, J. Garnier, C. Michel, V. Doya, and A. Picozzi, Phys. Rev. A 83, 033838 (2011).

16. M. Zhurahov, A. Bekker, B. Levit, R. Weill, and B. Fischer, Opt. Express 24, 6553 (2016).

17. M. Segev and D. Christodoulides, Spatial Solitons, S. Trillo and W. Torruellas, eds. (Springer, 2001).

18. S. A. Ponomarenko and G. P. Agrawal, Phys. Rev. E 69, 036604 (2004).

19. L. Mokhtarpour and S. A. Ponomarenko, Opt. Express 23, 30270 (2015).

20. A. Picozzi and J. Garnier, Phys. Rev. Lett. 107, 233901 (2011).

21. A. Picozzi, S. Pitois, and G. Millot, Phys. Rev. Lett. 101, 093901 (2008).

22. B. Kibler, C. Michel, A. Kudlinski, B. Barviau, G. Millot, and A. Picozzi, Phys. Rev. E 84, 066605 (2011).

23. G. Xu, J. Garnier, M. Conforti, and A. Picozzi, Opt. Lett. 39, 4192 (2014).

24. G. P. Agrawal, Nonlinear Fiber Optics, 5th ed. (Academic, 2013).

25. T. Hansson, E. Wallin, G. Brodin, and M. Marklund, J. Opt. Soc. Am. B 30, 1765 (2013)

26. L. G. Wright, S. Wabnitz, D. N. Christodoulides, and F. W. Wise, Phys. Rev. Lett. 115, 223902 (2015).

27. K. Krupa, A. Tonello, A. Barthélémy, V. Couderc, B. Shalaby, A. Bendahmane, G. Millot, and S. Wabnitz, Phys. Rev. Lett. 116, 183901 (2016).

28. S. Trillo and S. Wabnitz, J. Opt. Soc. Am. B 9, 1061 (1992).

29. E. A. Golovchenko and A. N. Pilipetskii, J. Opt. Soc. Am. B 11, 92 (1994).

30. S. L. Musher, A. M. Rubenchik, and V. E. Zakharov, Phys. Rep. 252, 177 (1995).

31. J. Garnier and A. Picozzi, Phys. Rev. A 81, 033831 (2010).

32. P. K. A. Wai and C. R. Menyuk, J. Lightwave Technol. 14, 148 (1996). 